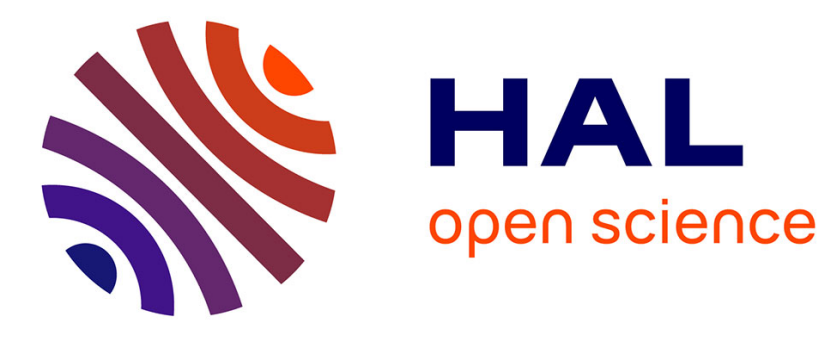

\title{
Foam clogging
}

Florence Rouyer, Benjamin Haffner, Nicolas Louvet, Yacine Khidas, Olivier

\section{Pitois}

\section{To cite this version:}

Florence Rouyer, Benjamin Haffner, Nicolas Louvet, Yacine Khidas, Olivier Pitois. Foam clogging. Soft Matter, 2014, 10 (36), pp.6990 - 6998. 10.1039/c4sm00496e . hal-01071753

\section{HAL Id: hal-01071753 \\ https://hal-enpc.archives-ouvertes.fr/hal-01071753}

Submitted on 6 Oct 2014

HAL is a multi-disciplinary open access archive for the deposit and dissemination of scientific research documents, whether they are published or not. The documents may come from teaching and research institutions in France or abroad, or from public or private research centers.
L'archive ouverte pluridisciplinaire HAL, est destinée au dépôt et à la diffusion de documents scientifiques de niveau recherche, publiés ou non, émanant des établissements d'enseignement et de recherche français ou étrangers, des laboratoires publics ou privés. 


\title{
Foam Clogging
}

\author{
F. Rouyer ${ }^{1}$, B. Haffner ${ }^{2}$, N. Louvet ${ }^{3}$, Y. Khidas ${ }^{1}$ and O. Pitois ${ }^{2}$ \\ ${ }^{1}$ Université Paris Est, Laboratoire Navier, UMR 8205 CNRS - École des Ponts ParisTech - IFSTTAR \\ 5 bd Descartes, 77454 Marne-la-Vallée Cedex 2, France \\ ${ }^{2}$ Université Paris Est, Laboratoire Navier, UMR 8205 CNRS - École des Ponts ParisTech-IFSTTAR \\ cité Descartes, 2 allée Kepler, 77420 Champs-sur-Marne, France \\ ${ }^{3}$ Université de Lorraine, LEMTA, UMR 7563, Vandoeuvre-lès-Nancy, F-54500, France
}

\begin{abstract}
:
To what extent do aqueous foams clog? Foam permeability is measured as a function of particle loading. The particle to bubble size ratio is allowed to increase when the particle to bubble number ratio is fixed. In addition to experiments performed on the foam scale, we investigate experimentally and numerically the hydrodynamic resistance of a single foam node loaded with one particle. It is shown that, with respect to their solid counterpart, foams clog more efficiently for two reasons: (i) the deformation of interfaces allows for larger particles to be incorporated within the interstitial network and (ii), the interfacial mobility contributes to lower the reduced permeability.
\end{abstract}

\section{Introduction:}

Permeability reduction caused by particle deposition in porous media, or clogging, is widely encountered in technological processes of solids extraction from suspensions (deep-bed filtration) [1], as well as in natural aquifers [2]. Clogging, which is intimately related to particle capture, is a complex phenomenon involving a large number of parameters that have been partially decoupled in experiments conducted on model systems, such as beds of solid spheres [3], sieves [4] or solid channels [5].

To a certain extent, aqueous foams can be considered as porous media, exhibiting fine liquid channels between gas bubbles. The main asset of foams is their capacity to adjust dynamically the channel size over several orders of magnitude, in response to changes in interstitial liquid flow conditions [6]. Moreover, foam channels can capture particles suspended in the liquid flowing through the gas bubbles. The capture mechanism has been shown to be controlled by the confinement parameter $\lambda$ that compares the particle size to the size $d_{C}$ of passage through 
constrictions in the interstitial network of the foam [7,8], the latter size being set by bubble size and gas fraction. In the absence of collective trapping, i.e. jamming of the confined concentrated particle suspension [9], the capture of particles is triggered as soon as $\lambda \gtrsim 1$. In contrast to their solid counterpart, aqueous foams can be loaded with large particles that deform the interstitial network which suggests strong clogging effect. On the other hand, the high mobility of bubble surface is known to increase significantly the liquid permeability of aqueous foams [10]. This specific interfacial behaviour is expected to maintain a significant level of liquid permeability through foam channels obstructed with solid particles. Therefore, a simple question arises: to what extent do aqueous foams clog? Beyond the fundamental aspect of this issue, liquid drainage reduction due to clogging has a beneficial effect on foam stability, which can be of particular interest for the numerous applications of foams.

In the present work, we determine the foam permeability reduction caused by particle loading, at the rate of one particle per foam node. In addition to experiments performed on the foam scale, we investigate experimentally and numerically the hydrodynamic resistance of a single foam node loaded with one particle.

\section{Single node experiment:}

The experimental setup used in this study has been previously described and more details can be found elsewhere [11]. We just recall that a Plateau border and the three adjoining films are formed on withdrawing a tripod from a reservoir containing the foaming solution. The latter contains $10 \mathrm{~g} / \mathrm{L}$ of TetradecylTrimethyl-Ammonium Bromide (TTAB) in distilled water. The density, the surface tension of the liquid/gas interface and shear viscosity of the bulk are respectively $\rho \simeq 1000 \mathrm{~kg} / \mathrm{m}^{3}, \sigma \simeq 38 \mathrm{mN} / \mathrm{m}$ and $\mu \simeq 1 \mathrm{mPa} . \mathrm{s}$. The height of the resulting Plateau border is approximately $5 \mathrm{~mm}$. The solution can be further delivered through the channel at a controlled flow rate $\mathrm{Q}$ when a pressure drop is imposed. A single foam node can be generated in releasing a small gas bubble from the reservoir [12].

Such a foam node was studied and the corresponding hydrodynamic resistance was measured [12]. Here, we follow the same measurement procedure excepted that a small glass particle is introduced in the node (see Fig. 1a) before measuring the pressure $\Delta P$ required to impose the liquid flow rate through the system. $\Delta P$ results from the association in series of the upper Plateau border $(\mathrm{Pb} 1)$, the node $(\mathrm{n})$, and the three lower Plateau borders $(\mathrm{Pb} 2)$, themselves in parallel association: $\Delta P=\Delta P_{1}+\Delta P_{n}+\Delta P_{2} . \Delta P_{1}$ and $\Delta P_{2}$ are determined from the geometry 
of the corresponding Plateau borders, as detailed in [12] and $\Delta P_{n}$ is deduced. We define the dimensionless resistance: $\tilde{R}_{n}=\left(r_{P b}^{3} / \mu\right)\left(\Delta P_{n} / Q\right)$, where $r_{P b}$ is the radius of curvature that characterizes the Plateau borders connected to the node (see also Fig. 3). Here, $r_{P b} \approx 1.2 \mathrm{~mm}$.

The main experimental error on $\tilde{R}_{n}$ is due to the strong contribution of $\Delta P_{1}$ to $\Delta P$, whose absolute error impacts directly $\Delta P_{n}$. In order to minimize this effect, we restrain our study to situations where the upper Plateau border $(\mathrm{Pb} 1)$ is not deformed by the particle, i.e. $\lambda \leq 2$ (two particle diameters were considered: 480 and $650 \mu \mathrm{m}$ ). In the following we will refer to the reduced resistance parameter, obtained by dividing the dimensionless resistance of a loaded node $\tilde{R}_{n}(\lambda)$ by the one corresponding to the empty node $\tilde{R}_{n 0}$.

\section{Experiments at the foam scale:}

\subsection{Preparation of particle-laden foams}

Samples are prepared from precursor liquid foam which is subsequently mixed with a granular suspension as previously presented in [9]. Using appropriate bubbling methods in a foaming solution (TTAB $10 \mathrm{~g} / \mathrm{L}$, glycerol, water), a foam with bubble diameter $D_{b}$ is made in a vertical column. For the present study bubble size has been varied within the range 450-800 $\mu \mathrm{m}$. For each sample, the relative deviation in bubble size is lower than $10 \%$. Liquid imbibition from the top of the column allows maintaining the liquid fraction at a constant value throughout the foam sample during the foam production. The foam is then pushed toward a T-junction where a suspension of polystyrene beads (diameter $d_{p}=80 \mu \mathrm{m}$ and $d_{p}=$ $140 \mu \mathrm{m})$ is injected. The liquid phase is the same for the foam and for the suspension; its density was matched with that of polystyrene $\left(\rho=1050 \mathrm{~kg} \cdot \mathrm{m}^{-3}\right)$ by adjusting the proportion of glycerol $(20 \% \mathrm{w} / \mathrm{w})$ and its bulk viscosity is $\mu \simeq 1.7 \mathrm{mPa} . \mathrm{s}$. The resulting gas and particle fractions, respectively $\phi$ and $\phi_{p}$, are set by the liquid fractions and the flow rates of injected foam and suspension. In the following, the gas fraction is set to $\phi=0.9$. This corresponds to wet foams in which the interstitial volume is mainly occupied by the nodes. The particle volume fraction in the interstitial suspension is $\varphi_{p}=\phi_{p} /(1-\phi)$. Our method has been found to produce homogeneous samples, characterized by well-distributed particles and bubbles, the size of the latter being preserved during the mixing step (an illustration of samples' quality is presented in Fig.1b). The laden foam is then continuously introduced in a 
rotating horizontal column used to compensate the effects of drainage during the preparation of the sample.

As we try to clog the foam by incorporating particles in the foam nodes, we focus on situations where the number of particles is equal to the number of foam nodes. This condition is fulfilled if $\varphi_{p} \equiv \varphi_{p, 1}=6 \phi d_{p}^{3} /(1-\phi) D_{b}^{3}$ (6 nodes per bubbles were considered). It is more appropriate to compare the size of the particles to the one that characterizes the foam channel network. This can be done in introducing the confinement parameter $\lambda=d_{p} / d_{c}=$ $C(\phi) d_{p} / D_{b}$ where $d_{c}$ is the size of passage through the network constrictions and $C(\phi)=$ $\left(1+0.57(1-\phi)^{0.27}\right) /\left(0.27 \sqrt{(1-\phi)}+3.17(1-\phi)^{2.75}\right)[7]$. Thus:

$$
\varphi_{p, 1}=\frac{6 \phi}{(1-\phi)}\left(\frac{C(\phi)}{\lambda}\right)^{-3}
$$

For $\phi=0.9$, eq. 1 reduces to $\varphi_{p, 1}=0.018 \lambda^{3}$. We investigate the effect of particle loading in foams within the $\lambda$-range $1.6-2.5$ by changing the bubble size, keeping constant the particle size as well as the gas fraction. The lower value is set by the particle retention properties of the foam. Indeed, it has been shown that for $\lambda \gtrsim 1.6$, particles are definitely trapped by the foam column, whereas particle retention is not complete within the range 1-1.6 [8]. For $\lambda=2.5$, the required particle volume fraction set by eq. 1 rises up to almost 0.3 . Going above this value would raise two issues: (i) the jamming of the particles could superimpose to the clogging issue we are looking for, (ii) difficulties are encountered with the present experimental setup to achieve high $\varphi_{p}$ values at relatively high $\lambda$ values.

\subsection{Permeability measurements}

Once the column is filled with the foamy suspension, it is turned to the vertical direction and the measurement of the free-drainage velocity starts. Note that with the present procedure, the starting point is a foam column with a uniform vertical gas fraction profile. Drainage is followed through the height $h(t)$ corresponding to the volume of suspension drained off at the bottom of the column. Such a measurement is plotted in Fig. 2 for particle-free and particleladen foams, showing a first stage characterized by a rapid linear increase of $h(t)$ followed by a slower evolution towards the equilibrium value. During the first regime, the volume of liquid draining out of the foam flows through foam areas that are not yet reached by the 
drainage front, i.e. areas where the gas fraction remains constant and equal to the initial value, $\phi=0.9$. This also applies to the particle volume fraction $\varphi_{p, 1}$ and $\lambda$. In this regime, the drainage velocity $V=d h / d t$ identifies to the Darcy velocity, i.e. $V=k \rho g / \mu$, where $k$ is the foam permeability $[6,10,13]$. In the following, we refer to the reduced foam permeability, i.e. $\tilde{k}=k\left(\varphi_{p, 1}\right) / k_{0}$, where $k_{0}$ is the permeability of the particle-free foam having the same parameters $\left(\phi, D_{b}\right)$ than the particle-laden foam.

\section{Numerical simulations:}

\subsection{Geometry of loaded and particle-free nodes}

The interstitial volume of foams is composed of liquid channels, the so-called Plateau borders, and their junctions, or nodes. The Plateau borders are formed by the merging of foam films when they intersect symmetrically three by three, and their cross-section is bounded by three tangentially connected circular arcs of radius $r_{P b}$ and angle $\pi / 3$. Nodes are formed when four Plateau borders join symmetrically at the centre of a tetrahedron, as depicted in Fig. 3. The complex geometry of nodes results from capillary law: the curvature of interfaces is imposed by the liquid/gas pressures according to the Young-Laplace equation. The precise shape of the node can be conveniently simulated using the Surface Evolver software [14], which is based on the principle that the equilibrium foam structure is such that its interfacial energy density is minimal. For the present study, we simulate a network element, i.e. a node connected to its four Plateau borders, representative of wet foams (see Fig. 3). As already said, in such conditions, the volume fraction of the Plateau borders is small compared to the volume fraction of nodes. Described in terms of length, the Plateau borders represent approximately $10 \%$ of the node-to-node distance in the foam network [13].

The solid particle is modelled by inserting within the node a body with a surface tension equal to 30 times the surface tension of liquid-air interface (see Fig. 4). Its volume is fixed to $v_{p}=\pi d_{p}^{3} / 6$. The complete volume of the loaded element, $v_{i}$, is kept constant so that the particle volume fraction $\varphi_{p}=v_{p} / v_{i}$ increases as the particle size increases.

We consider the confinement parameter $\lambda=d_{p} / d_{c}$, where $d_{c}=(2 / \sqrt{3}-1) r_{P b}$. Note that in the following $r_{P b}$ refers to the geometry of the particle-free element. We simulate geometries for $\lambda$ within the range 1.2-2.7. For small $\lambda$, the node geometry is not deformed by the particle 
which is free to explore a given set of positions within the interstitial volume (see Fig. 4-left). In contrast, for larger $\lambda$ the particle position becomes imposed at the center of the geometry thanks to capillary forces induced by interface deformations (see Fig. 4-right). This transition between non-deformed/deformed geometries has been found to occur at $\lambda \simeq 2$.

\subsection{Simulation of the liquid flow in nodes}

Geometries provided by the Surface Evolver software are imported in Comsol Multiphysics. Further meshing procedures were applied ('auto' extra-fine mesh was used and growth rate was imposed to be equal to 1.1 at liquid interface and 1.02 at the edges) in order to obtain geometries suitable for the simulation of liquid flow. As the real size of the geometries are of the order of $10^{-4} \mathrm{~m}$, the liquid velocity is of the order $10^{-3} \mathrm{~m} \cdot \mathrm{s}^{-1}$ and its density $\rho=$ $1000 \mathrm{~kg} \cdot \mathrm{m}^{-3}$ the Reynolds number is of the order $10^{-1}$. Therefore, Stokes equations are solved for real dimension of the geometries and for Newtonian fluid dynamical shear viscosity $\mu=1 \mathrm{mPa} . \mathrm{s}$ (note that calculations have shown insignificant deviations for results obtained from Navier-Stokes and Stokes). The interface in foam is known to be characterized by a certain degree of mobility [13], resulting in complex interfacial flows driven by the viscous drag from the bulk flow as well as stresses induced by surfactant exchanges with the bulk. In the absence of the complete description of these couplings for the foam channels, a pragmatic approach is to describe the behavior of this interface using an effective surface parameter. In a pioneer paper, Leonard and Lemlich [15] introduced the surface shear viscosity $\mu_{s}$. Within the last decade, $\mu_{s}$ was found to be useful to describe the drainage behavior of foams, at least in a semi-quantitative way [16], keeping in mind that this parameter should be considered as an effective surface parameter. Here, we follow this approach and we model the behavior of the node surface in terms of surface viscous stress associated to the parameter $\mu_{s}$. More precisely, the mobility is modeled by balancing the bulk viscous stress with the surface viscous stress at the liquid interface. The limit condition at the solid interface of the bead is zero velocity. Finally, it is assumed that liquid velocity vanishes at the junctions of liquid surfaces (edges). The partial derivative equations of vector velocity $\vec{v}$ write:

$$
\text { (in the liquid) } \Delta \vec{v}=\frac{1}{\mu} \vec{\nabla} p \quad \text { (Eq. 2a) }
$$




$$
\begin{aligned}
& \text { (at the liquid interface) } \mu(\vec{n} . \vec{\nabla}) \vec{v}=\mu_{S} \Delta_{S} \vec{v} \quad \text { (Eq. 2b) }
\end{aligned}
$$

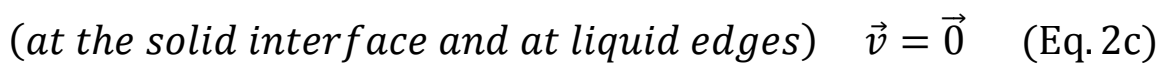

where $p$ is the liquid pressure (for stationary drainage conditions, the pressure gradient corresponds to gravity forces), $\Delta_{S}$ is the surface Laplacian and $\vec{n}$ is the unity vector normal to the surface pointing out of the geometry. The Boussinesq number compares the surface viscous stress to the bulk viscous stress: $B q=\mu_{s} / \mu r_{P b}$. For simulations, $\mu_{s}$ is varied from $10^{-8}$ up to $10^{-2} \mathrm{~kg} . \mathrm{s}^{-1}$ and, as the size $r_{P b}$ has been set to $0.581 \mathrm{~mm}, B q$ is varied within the range $2.10^{-1}-2.10^{4}$. Typical flows simulations are illustrated in figure 5 .

The hydrodynamic resistance of the node, $R_{n}=\Delta P / Q$, is determined when a pressure difference $\Delta P$ is imposed between the node ends. The liquid flow rate $Q$ is calculated by integrating the flow through the cross-sectional area of the node ends. Several configurations are studied: (1-3) 1 inlet and 3 outlets, (2-2) 2 inlets and 2 outlets and (3-1) 3 inlets and 1 outlet. Moreover, in situations where the particle has several possible positions, i.e. when $\lambda<2$, we consider always configurations where the particle is against an outlet. The normalized resistance is $\tilde{R}_{n}=\left(r_{P b}^{3} / \mu\right) \Delta P / Q$. In the following we will also refer to the reduced resistance: $\tilde{R}_{n} / \tilde{R}_{n 0}$, where $\tilde{R}_{n 0}$ is the resistance of the particle-free geometry.

\section{Results and discussion:}

We start with numerical results presented in Fig. 6. The effect of $B q$ on node resistance is illustrated in Fig. 6a for the particle-free node $\left(\tilde{R}_{n 0}\right)$ in configuration 1-3, i.e. the fluid enters the node by one inlet and leaves by three outlets, as well as a loaded node $\left(\tilde{R}_{n}\right)$ in configuration 1-3 with $\lambda=2$. In both cases the resistance is an increasing function of $B q$, and as expected, the resistance of the loaded node is larger than the corresponding particle-free node. This behavior has been observed for all studied geometries and configurations. Note that most of the resistance increase is observed within the $B q$ range 1-1000. In Fig 6b, the reduced resistance $\tilde{R}_{n} / \tilde{R}_{n 0}$ is plotted as a function of $B q$ for several $\lambda$ values in the range $2<\lambda<2.7$, i.e. which corresponds to situations where the node surface is deformed by the particle, the latter being centered with respect to the node geometry. $\tilde{R}_{n} / \widetilde{R}_{n 0}$ are decreasing functions of $B q$ due the fact that the relative increase of $\tilde{R}_{n 0}(B q)$ is stronger than the relative increase of $\tilde{R}_{n}(B q)$. The effect of $B q$ is all the more pronounced that $\lambda$ is high. Calculations 
(not presented in Fig. 6b) for configurations 2-2 and 3-1 have shown that the flow configuration has a very weak influence on $\tilde{R}_{n} / \widetilde{R}_{n 0}$ when the particle is centered. Fig. 6c shows a similar plot for $\lambda<2$. We have reported results for both 1-3 and 3-1 configurations (calculations for the 2-2 configuration - not reported in the figure - have provided values very close to the 1-3 configuration). Again, $\tilde{R}_{n} / \tilde{R}_{n 0}$ are decreasing functions of $B q$. As expected, within this $\lambda$ range $(\lambda<2)$, the effect of the flow configuration is very pronounced: in the 1-3 configuration, the particle clogs only one of the three outlets, so that the fluid flows through the two others free-outlets, whereas in the 3-1 configuration the unique outlet is clogged. We now focus on the 1-3 configuration, which is also the one corresponding to the single node experiment. In Fig. 7 the reduced resistance is plotted against $\lambda$ for $\lambda<2$. Numerical results are presented for several $B q$ values. Experimental data obtained from the single node experiment are also reported for comparison. The latter are in reasonable agreement with numerical $\tilde{R}_{n} / \tilde{R}_{n 0}$ values calculated for $B q=10$ and $B q=100$, whereas those calculated for $B q=1$ and $B q=1000$ deviate from experimental data. In order to say more about the relevant range of $B q$ values for comparison with experiments, data corresponding to the measured dimensionless resistance of the particle-free node [12] are reported in Fig. 6a. This shows that experimental values are consistent with numerical resistances calculated with $\mathrm{Bq}$ values within the range 10-100. Therefore, for both particle-free and loaded nodes, the relevant $B q$ range has to be set between 10 and 100. The corresponding values for $\mu_{s}$ are respectively $6.10^{-6}$ and $60.10^{-6} \mathrm{~kg} . \mathrm{s}^{-1}$. These values are at least two orders of magnitude larger than those generally accepted for Plateau borders geometries [16]. Note that $r_{P b}$ values are respectively 0.6 and $1.2 \mathrm{~mm}$ for the numerical and the experimental nodes so that a variation of $\mu_{s}$ by a factor 2 is expected between the two systems for a given $B q$ value. Obviously, this variation is contained within the bounds defined by $B q=10$ and $B q=100$. As already pointed out [17], $\mu_{s}$ should be thought in terms of effective parameter, whose value has to be adapted to flow conditions imposed at the liquid surface. Flows at node surface are known to differ significantly from those developed at Plateau border surface. In particular, significant surfactant adsorption/desorption phenomena are expected to reduce the surface mobility of nodes [18], which is in consistent with $\mu_{s}$ values provided by the present study. Although considering $\mu_{s}$ as an adaptive parameter is not fully satisfactory on the fundamental point of view, this approach has however provided some basic understanding of foam drainage [13] and the present work goes one step further in the evaluation of the relevance of $\mu_{s}$. Having identified the appropriate range of $\mu_{s}$ values to be used in the 
numerical simulation, we now turn to the results provided by the foam experiment. The size $r_{P b}$ in the studied foam are 4 to 6 times smaller than $r_{P b}$ used in the simulation. Although this variation should be accounted for when comparing experimental data to calculations, the expected effect is contained within the bounds defined by $B q=10$ and $B q=100$.

The results for the reduced permeability of particle-laden foams are presented in Fig. $8 . \tilde{k}$ decreases as a function of $\varphi_{p, 1}$ (we recall that $\varphi_{p, 1}$ is the particle volume fraction corresponding to one particle per foam node). This behavior due to particle loading is usually observed for solid porous media, such as filters for example. We will try to quantify the observed decrease for loaded foams with respect to their solid counterpart. First of all, we compare the experimental data to numerical results. As each foam node is loaded with one particle, the inverse of the reduced foam permeability, i.e. $k_{0} / k\left(\varphi_{p, 1}\right)$, is equal to the reduced node resistance $\tilde{R}_{n} / \widetilde{R}_{n 0}$. The comparison is presented in Fig. 9 as a function of $\lambda$. The agreement is reasonable using, for $\lambda<2$, the numerical values for the 3-1 configuration at $B q=10$ (upper bound) and the 1-3 configuration at $B q=100$ (lower bound). The whole set of data shows that the reduced node resistance increases up to 7 as $\lambda$ is close to 2.7. Whereas $\tilde{R}_{n} / \tilde{R}_{n 0}$ values corresponding to the node experiment are consistent with numerical values for the 1-3 configuration, values obtained from the foam experiment are between those for the 1-3 and the 3-1 configurations, which accounts for the averaging effect induced by foam (we recall that results for the 2-2 configuration are very close to those of the 1-3 configuration). At this stage, we have no rigorous way to calculate the average values from numerical data obtained with 1-3 and the 3-1 configurations, but in considering both upper and lower bounds of Fig. 9, experimental data could be described by a contribution of 50\% for each value. In order to carry on the comparison of the results, we report in Fig. 8 numerical data obtained for $\lambda>2$ (for which configurations 1-3, 2-2 and 3-1 are equivalent) for several values of $B q . B q=25$ is used to fit experimental results, $B q=1$ is used to show the limit of very mobile interfaces, and $B q=10^{4}$ is used to show the limit of non-mobile interfaces. We also resort to the Carman-Kozeny model [19] to estimate the permeability decrease. It is based on the specific surface area $A_{s}$, i.e. the surface area in contact the fluid divided by the volume $v_{t}$ of the porous medium. In foam, $v_{t}=v_{g a s} / \phi$, and when considering one foam bubble of diameter $D_{b}$ surrounded by the corresponding volume fraction of liquid: $v_{t}=s_{0} D_{b} / 6 \phi$, where $s_{0}=\pi D_{b}^{2}$. The specific surface area of the particle-free foam has been estimated to be $A_{s, \text { foam }}=3.88 / D_{b}$ for $\phi=0.9$ [20]. The additional specific surface area due 
the particles is $\left(6 \phi / D_{b}\right) S_{p} / s_{0}$, where $S_{p}$ is the surface area of the particles. As there are 6 nodes per foam bubble [13] and 1 particle per node for the present study, $S_{p} / s_{0}=6 d_{p}^{2} / D_{b}^{2}$. When $\lambda>2$, the node surface is deformed by the particle, as shown in Fig. 10b, and $A_{s, \text { foam }}$ is consequently modified. This evolution is determined from the Surface Evolver calculations and it is formulated in terms of a shape factor $\mathcal{F}_{n}(\lambda)$ which is the ratio between non-deformed and deformed node surface areas: $A_{s, \text { foam }}(\lambda)=3.88 \cdot \mathcal{F}_{n}(\lambda) / D_{b}$. Moreover, four contacts form between the particle and the node surface and the corresponding surface area from Surface Evolver can be written: $S_{p} \cdot \mathcal{F}_{f}(\lambda)$. Their negative contribution to the global specific surface area is $-2 \mathcal{F}_{f}(\lambda) \cdot\left(6 \phi / D_{b}\right) \cdot 6 d_{p}^{2} / D_{b}^{2}$. The evolution of the two shape factors $\left(\mathcal{F}_{n}\right.$ and $\mathcal{F}_{f}$ ) are plotted in Fig.10a as a function of $\lambda$. Then, according to the Carman-Kozeny model, the permeability of the loaded foam is $k=\left[(1-\phi)\left(1-\varphi_{p, 1}\right)\right]^{3} / C_{C K} A_{S}^{2}$, where the CarmanKozeny constant $C_{C K}$ has been shown to be equal to 5 for foams [20]. Finally, the dependence of the foam permeability on the particle volume fraction $\varphi_{p, 1}$ reads:

$$
\frac{k}{D_{b}^{2}}=\frac{(1-\phi)^{3}\left(1-\varphi_{p, 1}\right)^{3}}{C_{C K}\left[3.88 \mathcal{F}_{n}+\left(1-2 \mathcal{F}_{f}\right)\left(6^{4} \phi(1-\phi)^{2} \varphi_{p, 1}^{2}\right)^{1 / 3}\right]^{2}}
$$

Eq. 3 is plotted in Fig. 9. For $\lambda>2$ (equivalently $\varphi_{p, 1} \gtrsim 0.15$ ) the Carman-Kozeny model is close to the numerical values obtained for $B q=10^{4}$. Both curves describe the permeability reduction for foams with non-mobile interfaces. Note that the Carman-Kozeny curve is above experimental data within the full range of investigated $\lambda$ values. This clearly shows that interfacial mobility in foams emphasizes the effect of particle loading.

\section{Conclusion}

The reduction of permeability as a function particle loading has been investigated for foams incorporating one particle per foam node, and in allowing for the particle to bubble size ratio to increase. Both experimental and numerical results provide a good understanding of the mechanisms that set the permeability of such particle-laden foams. With respect to their solid counterpart, these foams clog more efficiently for two reasons: (i) the deformation of interfaces allows for larger particles to be incorporated within the interstitial network and (ii), the interfacial mobility contributes to lower the reduced permeability. The first effect owns to the way we have loaded the foams, i.e. each node contains one particle and the particle-to- 
node size ratio is increased. The second one is due to the mobility contrast between foam and particles surfaces and it is expected to apply whatever the loading configuration.

\section{Aknowledgements}

We thank D. Hautemayou and C. Mézière for technical support. We are sincerely grateful to Simon Cox for help in first steps with Surface Evolver. We gratefully acknowledge financial support from Agence Nationale de la Recherche (Grant No. ANR-13-RMNP-0003-01) and French Space Agency (convention CNES/70980).

\section{References}

[1] C. Tien, Granular Filtration of Aerosols and Hydrosols (Butterworths, Boston, 1989); L. J. Zeman and A. L. Zydney, Microfiltration and Ultrafiltration: Principles and Applications (Marcel Dekker, New York, 1996).

[2] L. M McDowell-Boyer, J. R. Hunt, N. Sitar, Water Resour. Res., 1986, 22, 1901-1921.

[3] C. Ghidaglia, L. de Arcangelis, J. Hinch, and E. Guazzelli, Phys. Rev. E 53, R3028 (1996)

[4] N. Roussel, Thi Lien Huong Nguyen and P. Coussot, Phys. Rev. Lett., 2007, 98, 114502.

[5] H. M. Wyss, D. L. Blair, J. F. Morris, H. A. Stone, and D. A. Weitz, Phys. Rev. E, 2006, 74, 061402; K. V. Sharp and R. J. Adrian, Microfluid. Nanofluid., 2005, 1, 376.

[6] S. A. Koehler, S. Hilgenfeldt, and H. A. Stone, Langmuir, 2000, 16, 6327.

[7] N. Louvet, R. Höhler and O. Pitois, Phys. Rev. E, 2010, 82, 041405.

[8] Y. Khidas, B. Haffner and O. Pitois, submitted to Soft Matter (http://arxiv.org/abs/1312.6511).

[9] B. Haffner, Y. Khidas, O. Pitois, Soft Matter, 2014 (DOI: 10.1039/C4SM00049H)

[10] E. Lorenceau, N. Louvet, F. Rouyer, and O. Pitois, Eur. Phys. J. E, 2009, 28, 293-304.

[11] O. Pitois, C. Fritz , and M. Vignes-Adler, J. Colloid Interface Sci., 2005, 282, 458-65.

[12] O. Pitois, N. Louvet, E. Lorenceau, and F. Rouyer, J. Colloid Interface Sci., 2008, 322, 675-77

[13] D. Weaire and S. Hutzler, The physics of foams (Oxford University Press, United Kingdom, 2000); I. Cantat, S. Cohen-Addad, F. Elias, F. Graner, R. Höhler, O. Pitois, F. Rouyer, and A. Saint-Jalmes, Foams - Structure and dynamics, Ed. S. Cox (Oxford University Press, United Kingdom, 2013).

[14] K. Brakke, Exp. Math, 1992, 1, 141.

[15] R. A. Leonard and R. Lemlich, AIChE J., 1965, 11, 18-25; R. A. Leonard and R. Lemlich, AIChE J., 1965, 11, 25-29.

[16] S. Cohen-Addad, R. Höhler, and O. Pitois, Annu. Rev. Fluid Mech., 2013, 45, 241-67.

[17] P. Stevenson, J. Colloid Interface Sci., 2005, 290, 603-610.

[18] O. Pitois, N. Louvet, and F. Rouyer, Eur. Phys. J. E, 2009, 30, 27-35.

[19] J. Kozeny, Ber. Wien Akad., 1927, 136, 271; P.C. Carman, Trans. Inst. Chem. Eng., 1937, 15, 150.

[20] O. Pitois, E. Lorenceau, N. Louvet, and F. Rouyer, Langmuir, 2009, 25, 97-100. 

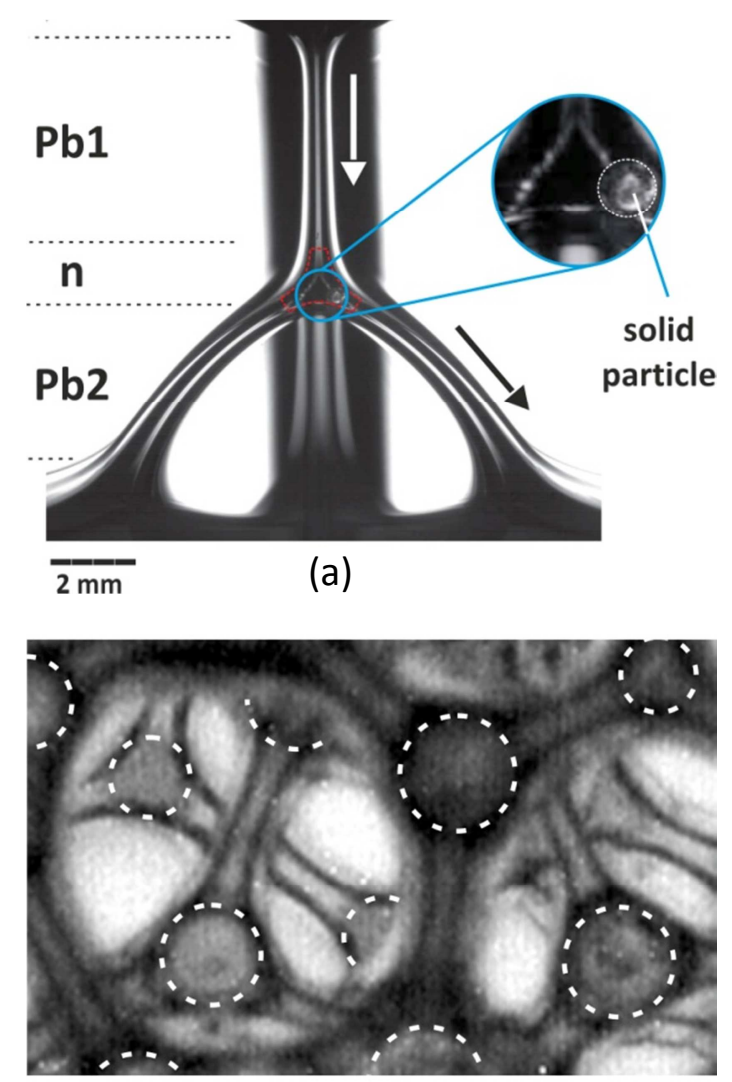

$100 \mu \mathrm{m}$

(b)

Fig 1: (a) Image of the node (the interior is delimited by the red dashed line) and the four Plateau borders studied in the so-called "single node experiment". The node contains one solid spherical bead of diameter $480 \mu \mathrm{m}$ located against one of the three outlets. Arrows show the direction of liquid flow. The measurement of the pressure required to impose a given liquid flow rate allows for the node hydrodynamic resistance to be determined (see [12] for more details about the experimental procedure). (b) Illustration of a particle-laden foams $\left(D_{b}=560 \mu \mathrm{m}, d_{p}=80 \mu \mathrm{m}\right)$. Note that in order to view inside the sample, the foam has partially drained. 


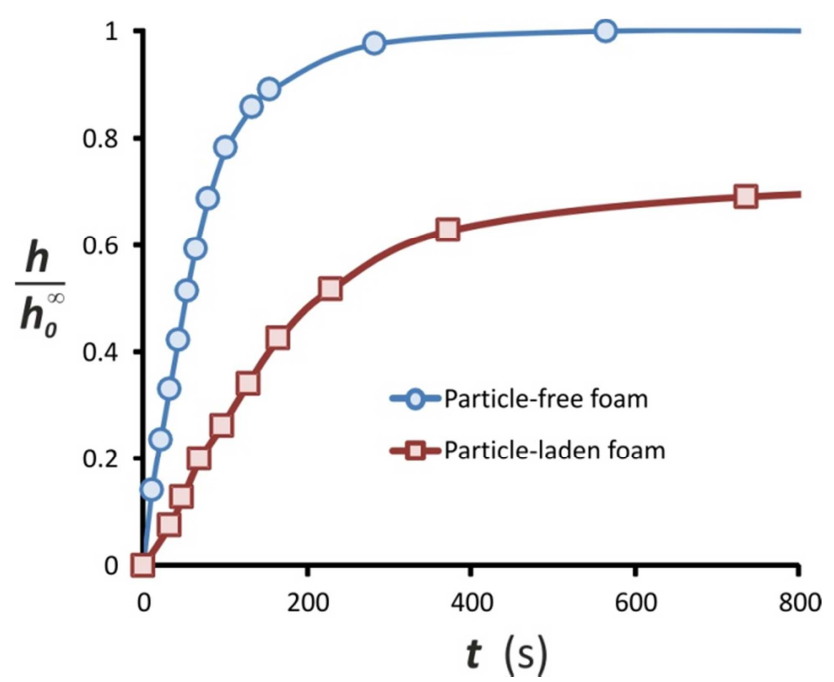

Fig 2: Temporal evolution of the reduced height of liquid drained out of the foam for particle-free $\left(D_{b}=\right.$ $510 \mu \mathrm{m}, \phi=0.9)$, and particle-laden foams $\left(D_{b}=510 \mu \mathrm{m}, \phi=0.9, d_{p}=80 \mu \mathrm{m}, \varphi_{p}=0.2\right) . h_{0}^{\infty}$ is the equilibrium value of $h(t)$ for the particle-free curve. 


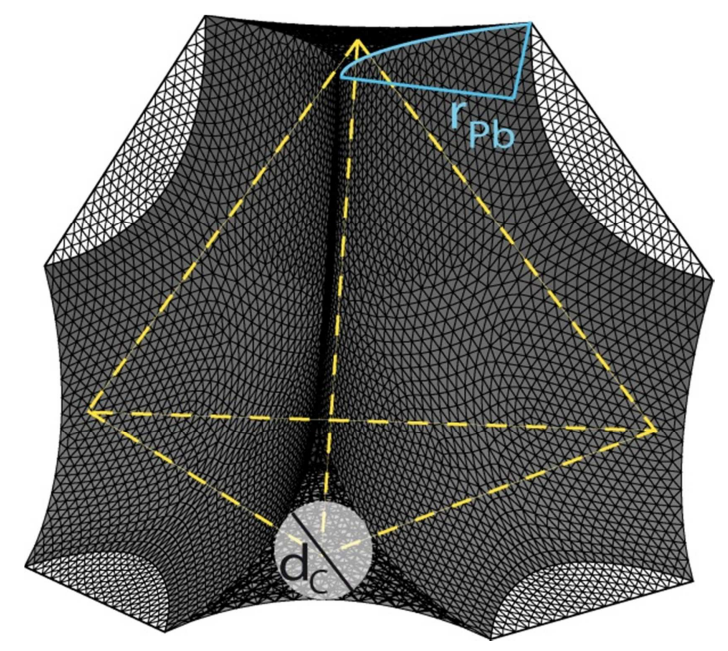

Fig. 3: Geometry of an empty node simulated by Surface Evolver. 

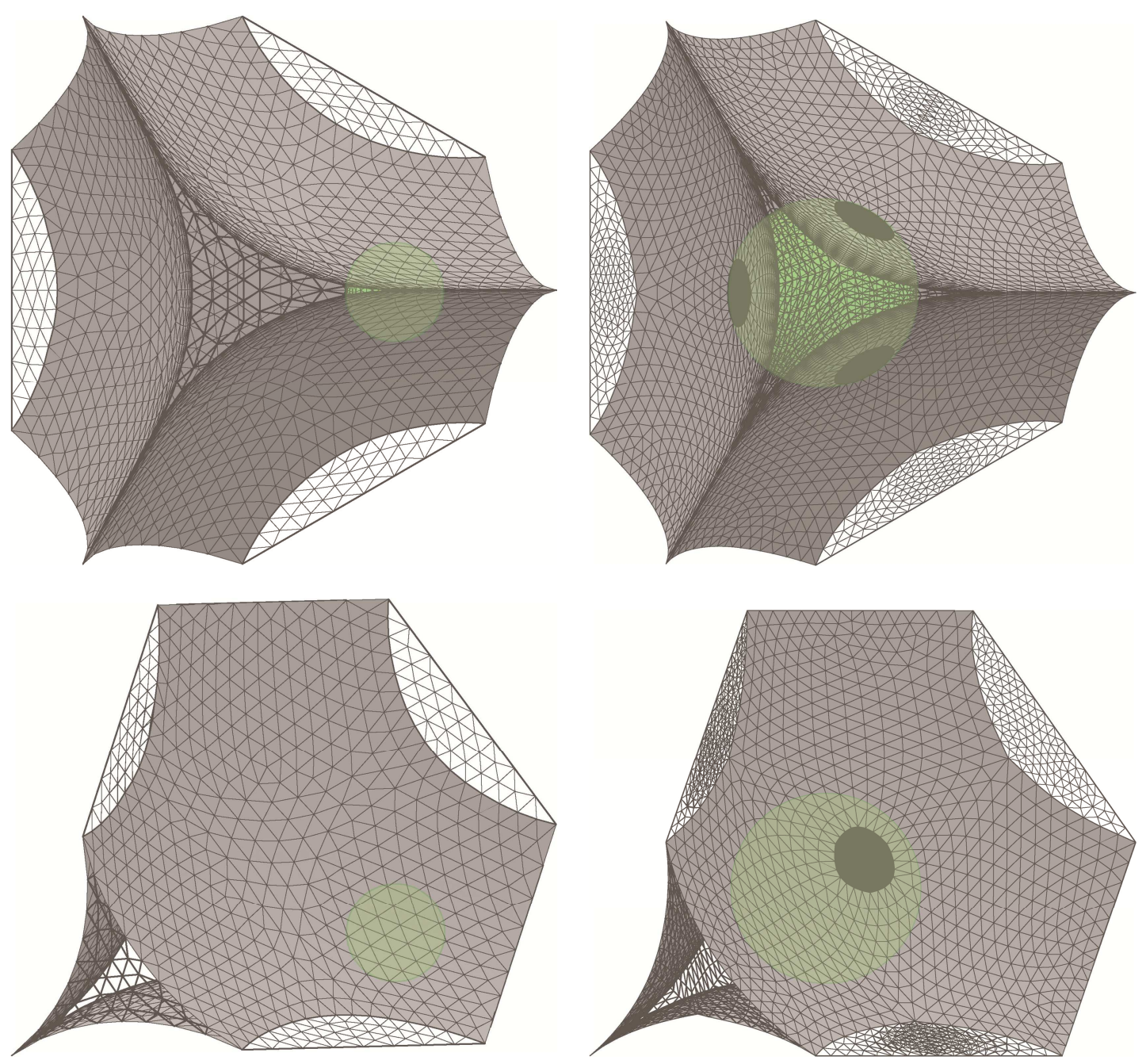

Fig. 4: Top and front views of clogged nodes simulated by Surface Evolver (left) $\lambda=1.20$ (right) $\lambda=2.30$. 


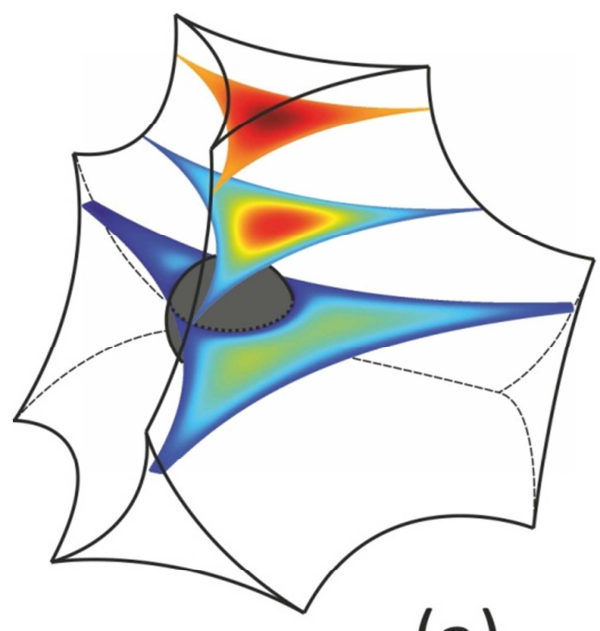

(a)

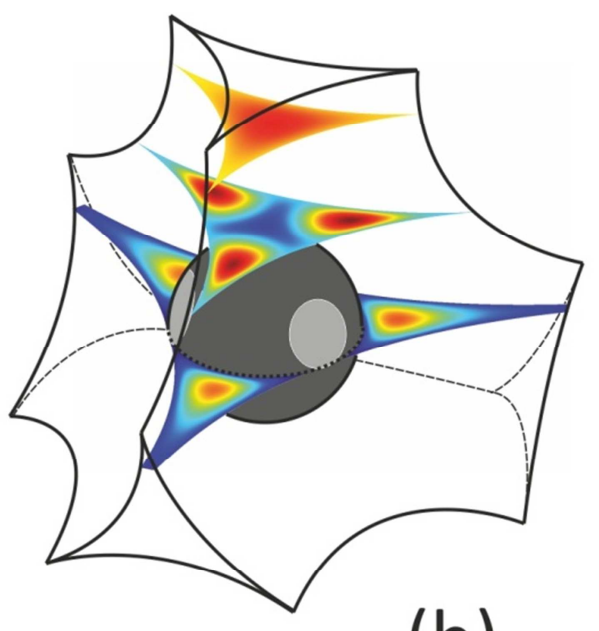

(b)
$\mathrm{V} / \mathrm{v}_{\max }$

1.0

0.8

0.6

0.4

0.2

Fig. 5: Comsol Multiphysics calculations of flows through nodes clogged with one particle with $\mu_{\mathrm{s}}=$ $10^{-5} \mathrm{~kg} \cdot \mathrm{s}^{-1}-\mathrm{Bq}=20$ : (a) $\lambda=1.57, \mathrm{v}_{\max }=0.875 \mathrm{~mm} / \mathrm{s}$; (b) $\lambda=2.30, \mathrm{v}_{\max }=0.308 \mathrm{~mm} / \mathrm{s}$. 

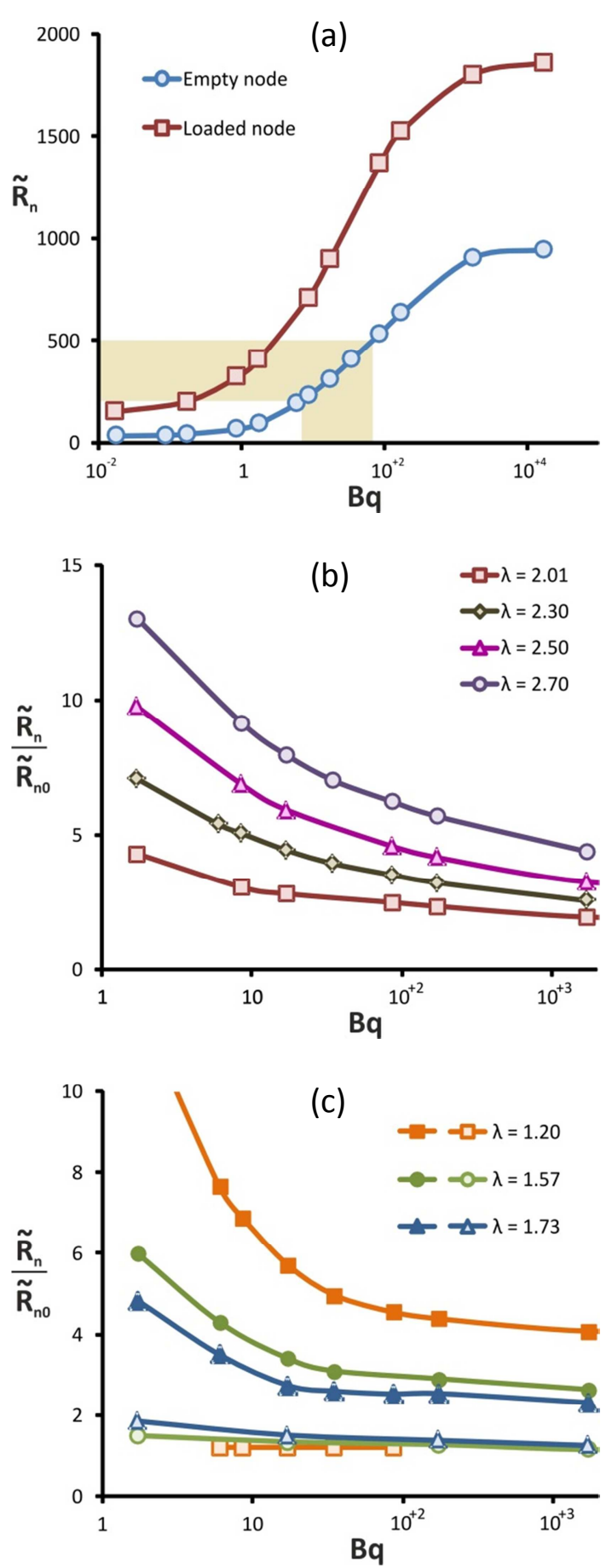

Fig 6: Hydrodynamic resistances of particle-free and loaded geometries obtained numerically when fluid enters the node by one inlet and leaves by three outlets (1-3 configuration). (a) Dimensionless resistance as a function of the Boussinesq number. The loaded node corresponds to $\lambda=2.01$. The shaded area highlights the node resistances obtained from the empty "single node experiment" [12] as well as the corresponding range of Boussinesq numbers. (b) Reduced resistances of loaded nodes (the resistance is divided by the one corresponding to the particle-free node) as a function of the Boussinesq number for several values of $\lambda$ in the range $\lambda>2$, i.e. the particle is centered and deforms the node surface. (c) Reduced resistances of loaded nodes as a function of the Boussinesq number for several values of $\lambda$ in the range $\lambda<2$. As the particle is small enough to have several positions, we present results obtained for the two relevant positions: $3-1$ configuration (filled symbols), i.e. the particle is against the outlet; 1-3configuration (open symbols), i.e. the particle is against one of the three outlets. 


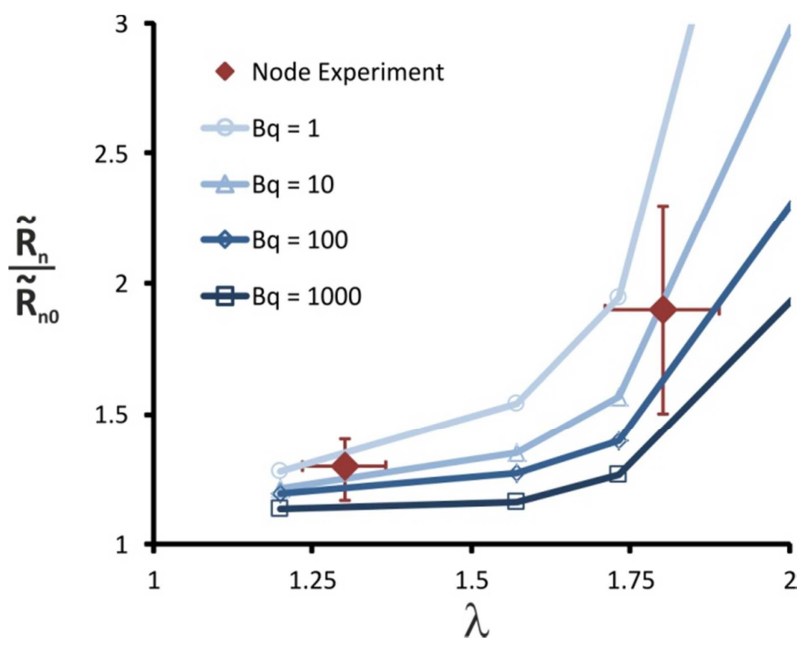

Fig 7: Numerical hydrodynamic resistances of loaded geometries compared to data provided by the "single node experiment" as a function of $\lambda$. Within that range of $\lambda$ values, the node surfaces are not deformed. Numerical calculations correspond to the experimental situation, i.e. 1-3 configuration, where fluid enters the node by one inlet and leaves by three outlets as the particle is against one of the three outlets. Several Boussinesq values are considered. 


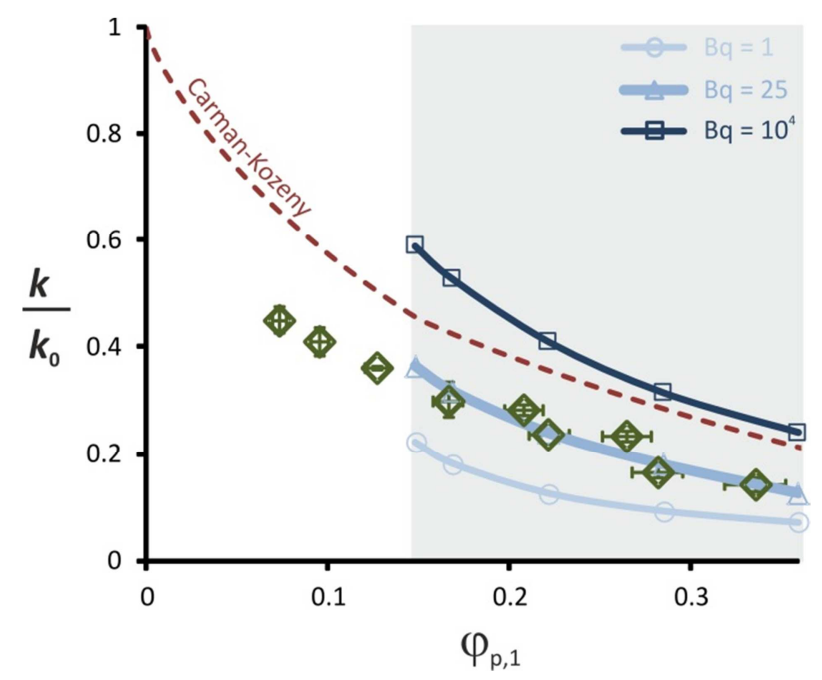

Fig 8: Experimental results for the reduced permeability of particle-laden foams as a function of the particle volume fraction. In the present loading configuration, each foam node is filled with one particle. Numerical results obtained for $B q=10^{4}, B q=25$ and $B q=1000$ are plotted for comparison $(\lambda>2)$ as well as eq. 3 . Interfacial mobility of foams intensifies the effect of particle loading on clogging. 


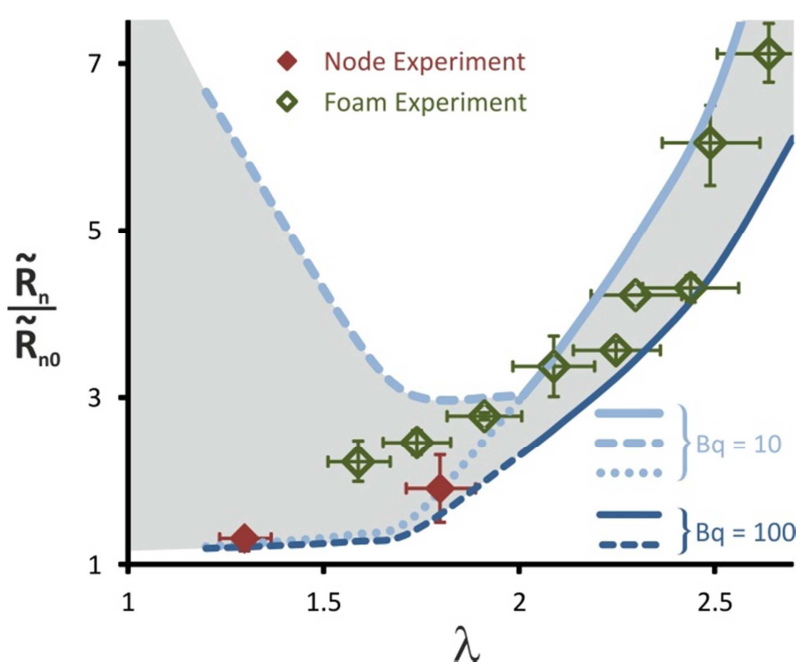

Fig 9: Numerical hydrodynamic resistances of loaded geometries compared to experimental data (single node and foam experiments) as a function of $\lambda$. Numerical results are presented for two Boussinesq numbers and two flow configurations. $B q=10: \lambda<2$ and 3-1 configuration (dashed line), $\lambda<2$ and 1-3 configuration (dotted line), $: \lambda>2$ (continuous line). $B q=100: \lambda<2$ and 1-3 configuration (dashed line), $: \lambda>2$ (continuous line). 

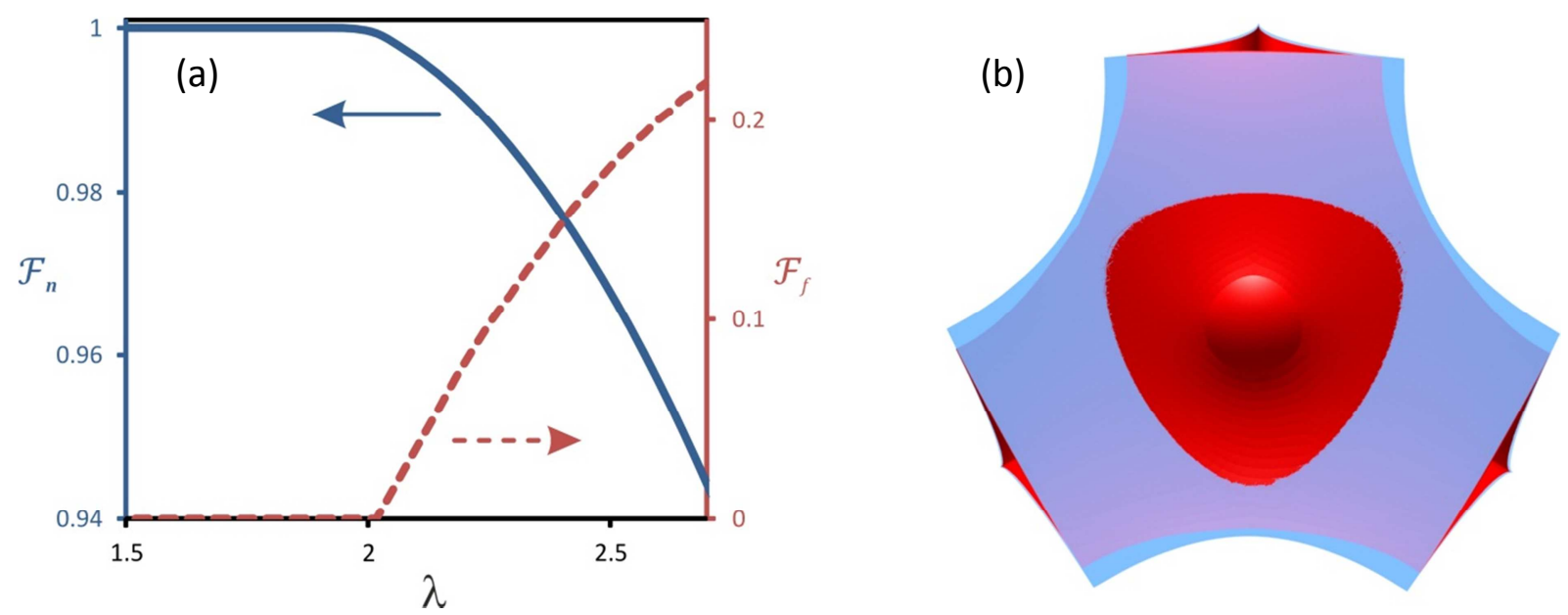

Fig 10: Surface Evolver calculations for the deformed node geometries: (a) $\lambda$-dependence of both the reduced node surface area $\left(\mathcal{F}_{n}\right)$ and the reduced contact area $\left(\mathcal{F}_{f}\right)$ between the particle and the node surface. (b) Superposition of the deformed (red) node surface $(\lambda=2.7)$ and non-deformed (blue) node surface. 